\title{
Educación para la ciudadanía en la era de las wikis
}

\author{
Education for Citizens \\ in the Wiki Era
}

\begin{abstract}
Gabriel J. Lotero-Echeverri (Colombia)
Comunicador Social, magíster en Mediación. Docente de la Fundación Universitaria Luis Amigó. Miembro del Grupo de Investigación Urbanitas (Medellín, Colombia).Correo electrónico: galotero@gmail.com

Fundación Universitaria Luis Amigó
\end{abstract}

\begin{abstract}
Resumen
El presente artículo tiene como propósito la revisión de los discursos optimistas sobre el acceso a las tecnologías de la información y la comunicación (TIC), los cuales, en ocasiones, no permiten considerar la apropiación social como un proceso complejo y multidimensional que representa un desafío para los diferentes actores del campo de la comunicación. Se destaca la necesidad de un abordaje crítico de los procesos de alfabetización mediática frente al reconocimiento de
\end{abstract}

\begin{abstract}
This article presents the revision of optimistic discourses on the access to the Information and Communications Technology (ICT), which, sometimes, do not allow considering social appropriation as a complex and multi-dimensional process that represents a challenge to the different actors on the field of communication. We highlight the need for a critical approach with respect to the process of media literacy and the recognition of the social appropriation of ICTs as an important
\end{abstract}

Para citar este artículo / to CIte thIS ARTICle

Lotero-Echeveri, G. J. (2014). Educación para la ciudadanía en la era de las wikis. Poliantea, 10(19), pp. 199-212. 
la apropiación social de las TIC como un factor constitutivo importante en la sociedad contemporánea, caracterizada como sociedad de la información.

Palabras clave: ciudadanía, comunicación digital, TIC, educación. factor that makes part of society nowadays, understood as information society.

Keywords: Citizenship, digital communication, ICT, education. 


\title{
Educación para la ciudadanía en la era de las wikis
}

\author{
Education for Citizens \\ in the Wiki Era
}

\begin{abstract}
Gabriel J. Lotero-Echeverri (Colombia)
Comunicador Social, magíster en Mediación. Docente de la Fundación Universitaria Luis Amigó. Miembro del Grupo de Investigación Urbanitas (Medellin, Colombia). Correo electrónico: galotero@gmail.com

Fundación Universitaria Luis Amigó
\end{abstract}

\section{Introducción}

Un discurso que es predominante en la actualidad es el que afirma que el acceso a internet representa una nueva forma de ciudadanía más activa y un sistema democrático participativo. Este discurso forma parte de las agendas y bienintencionadas declaraciones de organismos multilaterales, como la Unesco o la Organización de Estados Iberoamericanos tanto como de los gobiernos desde el nivel local hasta nacional.

Se trata de un enfoque ciberoptimista (Cardenal y Batlle, 2006) que imagina una sociedad más participativa como resultado automático del mayor acceso a la información y a herramientas digitales interactivas que hacen posible que la ciudadanía se exprese de manera directa.

Una postura optimista que afirma que disponer de acceso a herramientas interactivas representa la oportunidad para asumir una cultura política activa hacia formas más directas de democracia, propias de la llamada democracia deliberativa.

En este artículo se quiere problematizar dicha relación causal directa, casi automática, en la que incurren muchos discursos dominantes, la cual puede considerarse como idealista, al desconocer condiciones estructurales propias 
del contexto iberoamericano en general y colombiano en particular. Para lo cual se revisa una postura crítica que permita dudar de los efectos inmediatos del acceso a las herramientas tecnológicas en la cultura política de las personas.

\section{Objetivo}

Analizar la problemática relación entre la deliberación y las plataformas interactivas al servicio del ciberperiodismo, desde el enfoque de la educomunicación, con el fin de fomentar la reflexión acerca de los papeles de los actores involucrados en procesos educativos, en la escuela (como educación formal), en la apropiación social de las tecnologías de la información y la comunicación (TIC), al servicio de la ciudadanía.

\section{Metodología}

Estudio exploratorio cualitativo. El trabajo se aborda desde un nivel exploratorio, a partir de una revisión documental, acorde con el planteamiento del objeto de estudio y con los conceptos claves de la investigación.

\section{Estado de la cuestión}

Necesidad de una crítica de

\section{los discursos dominantes}

El discurso sobre los poderosos y benéficos efectos de las TIC en la ciudadanía puede leerse en la misma línea ideológica de los discursos dominantes del desarrollo (Sen, 2000), visto como único camino de progreso al cual están destinados los llamados países subdesarrollados (o atrasados) y que ha servido para legitimar tantos sacrificios y recortes para el bienestar de los pueblos del Sur (desde el Consenso de Washington a nuestro días, por ejemplo en el sur de Europa se siguen aplicando los recortes a los presupuestos sociales bajo receta del Fondo Monetario Internacional [FMI]). Este discurso ha sido problematizado desde líneas de pensamiento alternativas, poscoloniales, posdesarrollistas y altermundistas (Aparici, 2008).

También el discurso sobre el fortalecimiento de la ciudadanía y la consolidación de formas de democracia participativa gracias al uso de las TIC es dominante en el sentido en que orienta la construcción de políticas públicas y justifica la toma de decisiones sobre lo público y lo político, desde los gobiernos locales hasta los nacionales, los cuales se 
complacen en seguir los lineamientos de organismos multilaterales que los invitan a dar prioridad al acceso a las TIC de toda la población y la superación de la llamada brecha digital, la cual ha venido a complejizar la estructural brecha social que padecen los países latinoamericanos, entre los cuales Colombia siempre se destaca entre los más desiguales de una de las regiones más desiguales del planeta (PNUD, 2010).

En este artículo no se pretende negar la importancia del acceso a las TIC para todos y todas. Tan solo se busca reconocer otros puntos de vista que critican un exceso de optimismo, el cual desconoce las condiciones que deben intervenirse si la sociedad en su conjunto quiere ser más justa.

En este sentido, se justifica insistir en enfoques, como el de la educomunicación, desde el cual se analizan los procesos formativos que todo individuo debe tener a su alcance para poder hacer un mejor consumo de la superabundancia de información $\mathrm{y}$ de producciones de las industrias culturales, en el contexto de la sociedad contemporánea, caracterizada como sociedad de la información (Castells, 1996).

En esta reflexión, es necesario reconocer unos referentes como antecedentes en el campo de interés: el debate de la relación dinámica educación-comunicación, la revisión de fundamentos de ciudadanía y de educación para la ciudadanía y el reconocimiento de la situación actual del ciberperiodismo y de los entornos digitales interactivos.

\section{Las brechas digitales: cuestión de oportunidades}

Las TIC no están al alcance de todos y todas. El acceso a ellas es una meta central en las políticas del Gobierno colombiano. En Colombia, se estima que 8 de cada 10 personas, de entre 15 y 55 años, que habitan en ciudades colombianas de más de 200000 habitantes, utilizan internet (según encuesta financiada por el Ministerio de Tecnologías de la Información y las Comunicaciones [MinTIC], realizada en hogares de 13 ciudades, a 1005 personas, con un margen de error de $4 \%$ ). El $71 \%$ de los consultados acceden desde casa, $20 \%$ desde un café internet, $15 \%$ desde el trabajo y $8 \%$ desde la institución educativa, según datos de octubre de 2012 (Colombia, MinTIC e Ipsos Napoleón Franco, 2012). El reciente aumento se debe, en gran medida, al repunte de las conexiones a redes 
celulares, que ya superan $30 \%$ del total de suscripciones.

Esta brecha digital de acceso está imbricada con los altos índices de pobreza, miseria y desigualdad social, que estructural e históricamente padece Colombia. En este sentido, vale la pena destacar que quienes se encuentran en situación de exclusión y vulnerabilidad social actualmente padecen una nueva forma de analfabetismo: el tecnológico, el cual refuerza el círculo vicioso de la pobreza (Eclac, 2013).

La segunda amenaza la constituye otro tipo de brecha digital. Es necesario reconocer la importancia de la brecha de acceso, pero también una brecha de uso, que viene condicionada por las capacidades con las cuales cuenta el ciudadano para aprovechar adecuadamente el acceso a las TIC (Sunkel, Trucco y Möller, 2011).

En este sentido, cuando la información se ha extendido como un recurso superabundante, el propósito educativo debe ser no tanto la transmisión y memorización, propias de modelos tradicionales caracterizados como bancarios (Freire, 1983), sino una formación que permita que la persona tenga criterio propio, capacidad de análisis y de interpretación (Moacir y Gómez, 2003).
$\mathrm{Al}$ respecto, el sistema educativo colombiano tiene una deuda de calidad y no solo de cupos ofrecidos. De manera recurrente, los estudiantes colombianos se ubican en los últimos lugares de las pruebas PISA, en todos los componentes, sobre todo en comprensión de lectura (Semana, 2013). No es que estas pruebas tengan la última palabra, pero arrojan datos que se deben tener en cuenta, principalmente cuando las metas del Gobierno están ligadas solamente a la cobertura y no a la calidad.

Algunos autores insisten en que existe una brecha digital que puede ser más difícil de superar: la llamada tercera brecha digital (Fernández del Moral, 2012). Esta brecha hace referencia a la distancia que se genera entre quienes recurren a fuentes de información especializadas y verificables por las cuales circula el conocimiento experto y complejo, por una parte, mientras que otras personas acceden (tal vez la mayoría), debido a sus gustos e intereses, a fuentes de información informales, como la mayoría de los sitios de internet, por los cuales circula el conocimiento vulgar ${ }^{1}$.

1 Según la segunda acepción que presenta el Diccionario de la RAE: "Común o gene- 
Entonces no solamente es necesario garantizar el acceso a las TIC. Hay que analizar los usos que las personas dan a estas herramientas, más allá del entretenimiento.

Algunos autores critican la explicación individual de los gustos e intereses como si solo surgieran espontáneamente y hablan de preferencias adaptativas, para invitar a la reflexión en cuanto a en qué medida las aspiraciones, los gustos y los proyectos de comunidades y personas son el resultado de procesos educativos de larga duración, y que en muchas ocasiones se trata de falta de oportunidades (Pereira, 2009).

Por lo tanto, de alguna manera, algo falla cuando, en un mundo como el contemporáneo, los principales usos de internet son los resultados de los torneos deportivos o videos virales musicales surcoreanos; es como si aprendiéramos a desear mal (como afirmaba Estanislao Zuleta [2000], en su Elogio de la dificultad). Y la escuela tiene un papel que asumir en este problema.

ral, por contraposición a especial o técnico" (hitp://lema.rae.es/drae/? val=vulgar).

\section{Importancia de la dinámica} comunicación-educación

Con Freire, reconocemos:

la esperanza está en la raíz de la inconclusión de los hombres, a partir de la cual se mueven en permanente búsqueda $[. .$.$] La desesperanza$ es una forma de silenciar, de negar el mundo, de huir de él. La deshumanización que resulta del "orden injusto", no puede ser razón para la pérdida de la esperanza, por el contrario, debe ser motivo de una mayor esperanza, la que conduce a la búsqueda incesante de la instauración de la humanidad negada en la injusticia (1983, p. 111).

Se trata de una esperanza que no cae en la ingenuidad de pensar que con acceder a las TIC se superan las brechas sociales o se mejora la calidad del sistema democrático.

Desde la escuela debemos reflexionar en torno a la pregunta por la relación existente entre los procesos de apropiación social de las TIC y las nuevas formas de ejercer la ciudadanía potenciadas por escenarios virtuales, las cuales pueden rastrearse internacionalmente en movimientos sociales de gran repercusión, como las primaveras árabes, los movimientos 
de indignados y diversos mecanismos para visibilizar problemáticas relacionadas con violaciones a los derechos humanos $y$, a la vez, propiciar la denuncia y la exigibilidad ante los Gobiernos y entidades internacionales de todo el planeta.

La Unesco (1984) señala que los medios masivos de comunicación representan un desafío para la escuela, entendida como educación formal en sus distintos niveles, si se parte del reconocimiento de su innegable influencia en la vida cotidiana de nuestros estudiantes, sobre la sociedad en general, como fuente de información, como referente de valores y de modelos.

Lo que se afirma es que las personas están sometidas a una avalancha informativa sin precedentes, pero que el "consumo diario de medios de comunicación no es garantía de competencia audiovisual" (Aguaded-Gómez y Pérez-Rodríguez, 2012, p. 28).

Ante este desafío, diversos autores han señalado la necesidad de contribuir desde la escuela en procesos de alfabetización mediática, los cuales fortalezcan la formación de audiencias críticas, en sintonía con los lineamientos de la educación para la ciudadanía.
El valor de la educación en alfabetización mediática está en ayudar a los estudiantes a desarrollar su conocimiento y su comprensión de cómo operan los medios, cómo construyen su contenido, cómo pueden ser usados y cómo evaluar la información que plantean (Wilson y Duncan, 2009, p. 107).

De esta manera, nuestros estudiantes pueden comprender que los medios construyen sus agendas, encuadran la información, abordan determinadas fuentes de manera privilegiada y nombran el mundo con categorías dicotómicas que suelen poner énfasis en lo que nos separa y no en lo que nos une como ciudadanos del mundo (Cortina, 1997).

\section{Discusión de resultados}

Ante las condiciones que imponen los discursos de los grandes conglomerados de medios de comunicación, los movimientos sociales acuden a estrategias de comunicación alternativa, por fuera de las limitaciones de los discursos dominantes de los poderes establecidos tanto económicos como políticos, que suelen actuar como hermanos siameses.

Las acciones de comunicación alternativa, en Latinoamérica, 
encuentran un acumulado valioso en la perspectiva de la educación popular de Freire (1983). En la región existen experiencias de esta naturaleza como estrategias de apoyo para los movimientos sociales, que han encontrado en la radio un medio eficaz, así como en todo lo que permita la rica creatividad $^{2}$ para aprovechar todos los recursos de comunicación disponibles (Aparici y Torrent, 2010).

Esto no quiere decir que se desconozca que actualmente la comunicación alternativa es potenciada por las TIC, lo cual se evidencia en la importancia de medios digitales en red al servicio del llamado movimiento altermundista, de los movimientos sociales, los indignados, entre otros.

Estas experiencias de comunicación alternativa, también conocidas como periodismo ciudadano, se articulan en las plataformas electrónicas de los medios periodísticos, que les sirven de caja de resonancia y que han integrado espacios para que los usuarios interactúen, denuncien, opinen. Si algo caracteriza el ciberperiodismo,

2 Existen alternativas antes y después de internet, ligadas a la creatividad y al saber popular. Para superar barreras a la alfabetización incluso sirve un burro, como en la experiencia del biblioburro, que le permite a comunidades apartadas intercambiar libros (Ospina, 2012). es la superación de un modelo unidireccional, al permitir distintos niveles de interacción a los usuarios, que ya no se conciben solo como consumidores, sino también como productores de contenido (Franco, 2008).

Este modelo de comunicación, basado en la interacción, es pensado desde conceptos como el prosumidor, vislumbrado en los estudios sociológicos de Toffler (1981), para explicar la tendencia de los usuarios o destinatarios a superar un enfoque de recepción para ser también productores, en una dinámica en la cual se van intercalando ambos papeles.

Estos cambios en las rutinas de consumo se posicionan principalmente en las generaciones más jóvenes, de acuerdo con estudios como los de Noguera (2010).

Considerando las condiciones de los públicos a los cuales se pretende llegar, el oficio del periodismo se transforma tanto de forma como de fondo. El periodismo digital, o ciberperiodismo, describe las transformaciones del oficio del periodismo hecho para internet. El ciberperiodismo aprovecha el ciberespacio para todo el proceso de elaboración de textos, desde la investigación, la documentación y, principalmente, para la publicación de contenidos 
periodísticos pensados para un nuevo tipo de medio de comunicación, determinado por la transmedialidad, la convergencia de soportes, la integración de herramientas interactivas y la hipertextualidad (Scolari, 2008; Franco, 2008).

Existen críticas importantes a la postura optimista, que señalan que la participación de redes sociales no significa que las personas incrementen su exposición e involucramiento en discusiones sobre lo público y lo político, ya que se presenta una dinámica similar a la descrita en la teoría de la "espiral del silencio", en la cual las personas se callan sus pensamientos y opiniones si saben que contradicen a la mayoría de aquellos con quienes se relaciona en la red social, a la manada en la cual aspira a seguir siendo aceptado como uno más ( $E l$ Tiempo, 2014).

La educación para la ciudadanía se revaloriza ante la persistente apatía hacia lo público y lo político. En la debilidad de la cultura política en Colombia, inciden los altos índices de corrupción y la falta de transparencia como amenazas a la gobernabilidad (WVS, 2012; Medellín cómo vamos, 2012).

La encuesta mundial de valores 2012 (WVS, 2012) confirma el escaso interés de los ciudadanos colombianos en la política y la baja confianza en dos instituciones centrales del sistema: los partidos políticos y el Congreso. A pesar del histórico bipartidismo, una tercera parte de los consultados no simpatiza con ningún partido. Para la mayoría, se confirma la concepción de participación solamente como votar en las elecciones nacionales (67\%) y regionales (64\%).

\section{Consideraciones finales}

Tener acceso a internet, disponer de herramientas interactivas o de la posibilidad de publicar en blogs, wikis o redes sociales no es suficiente. Hace falta un proceso de concientización y de empoderamiento, como objetivos de intervenciones educativas transversales en formación ciudadana.

$\mathrm{Al}$ respecto de estas potencialidades de las TIC, Cardenal y Batlle (2006) critican que el ciberoptimismo es populista porque desconoce las condiciones de la estructura social y del campo de la política. Afirman que una postura populista se basa en dos argumentos: el primero consiste en asegurar que un mayor acceso a la información tiene efectos directos en el aumento de la participación política, en este caso, el problema es ignorar los 
costos (incluso conocimientos y tiempo) de acceso y procesamiento de la información; y el segundo argumento consiste en asegurar que las TIC hacen posible la democracia directa, el problema en este caso es desconocer el poder de los intermediarios (grandes medios de comunicación y partidos políticos). Por ejemplo, recientemente, ante la abdicación del rey borbónico, las mayorías parlamentarias no escucharon las múltiples voces que exigían un referendo popular que permitiera que la ciudadanía decidiera si quería la continuidad de la institución monárquica. Y los medios con mayor prestigio y audiencia (Ramos, 2013) dedicaron gran parte de su espacio a una agenda promonarquía, adornada con el glamur de doña Letizia.

En ambientes de polarización y unanimismo, propios del llamado pensamiento único dominante del cual habla Ramonet (1997), la crítica es recibida como un ataque o como manifestación de resentimiento. Quienes ven la realidad como una serie de oposiciones dicotómicas clasifican a quien critica una postura optimista como "apocalíptico" (a la manera del clásico Apocalípticos e integrados, de Umberto Eco, 1998). En el presente artíuclo, se invita a buscar una postura que vaya más allá de una "integración" de manera irreflexiva en la celebración del acceso a las TIC como la panacea a la débil cultura política colombiana.

No por ello se desconoce que las TIC son un recurso importante y que debe formar parte de las oportunidades que se han garantizar a cada persona para que sea agente de su propio destino, construya el tipo de vida que tenga razones para valorar (Sen, 2000) y pueda ejercer su condición ciudadana, acceder a información, participar en la discusión pública orientada a la búsqueda del bien común.

\section{Referencias bibliográficas}

Aguaded-Gómez, I. y Pérez-Rodríguez, M. A. (2012). Estrategias para la alfabetización mediática: competencias audiovisuales y ciudadanía en Andalucía. New Approaches in Educational Research, 1(1), 25-30.

Aparici, R. (2008). Educación para la comunicación en tiempos de neoliberalismo. Recuperado de http:// comunicacioneducativayculturapopular.blogspot.com/2007/01/bibliografa-de-la-asignatura.html 
Aparici, R. y Torrent, J. (2010). Educomunicación: participación ciudadana y creatividad. Recuperado de http:// www.1minutoxmisderechos.org.ar/ arch/EDUCOMUNICACION.pdf

Cardenal, A. S. y Batlle, A. (2009). La utopía virtual: una crítica al ciberoptimismo desde la teoría de la elección racional. IDP. Revista de Internet, Derecho y Politica, 3.

Castells, M. (1996). La era de la información: economía, sociedad y cultura (vol. I). Madrid: Alianza.

Colombia, Ministerio de Tecnologías de la Información y las Comunicaciones e Ipsos Napoleón Franco (2012). Encuesta de consumo digital en Colombia 2012. Recuperado de http:// es.slideshare.net/DiegoMolanoVega/ encuesta-de-consumo-digital

Cortina, A. (1997). Ciudadanos del mundo: hacia una teoría de la ciudadanía. Madrid: Alianza.

ECLAC (2013) Observatorio para la sociedad de la información en Latinoamérica y el Caribe. Recuperado de http://websie.eclac.cl/scripts/ broker.exe
Eco, U. (1998). Apocalípticos e integrados. Barcelona: Lumen.

El Tiempo (2014, 29 de agosto). Internet, una espiral que está acabando con el debate. Recuperado de http:// www.eltiempo.com/tecnosfera/novedades-tecnologia/internet-acabandocon-el-debate-dice-estudio/14454795

Fernández del Moral, J. (2012). La tercera y definitiva brecha digital. Telos: Cuadernos de Comunicación e Innovación, 91, 6-8.

Franco, G. (2008). Cómo escribir para la web. University of Texas: Knight Center of Journalism.

Freire, P. (1983). Pedagogía del oprimido. Madrid: Siglo XXI.

Medellín cómo vamos (2012). Participación ciudadana. Recuperado de http://www.medellincomovamos.org/ participaci-n-ciudadana

Moacir, M. y Gómez, L. (2003). Lecciones de Paulo Freire, cruzando fronteras: experiencias que se completan. Buenos Aires: Clacso. Recuperado de http://bibliotecavirtual.clacso.org.ar/ ar/libros/freire/freire.html

Noguera Vivo, J. M. (2010). Redes sociales como paradigma periodístico. 
Medios españoles en Facebook. Revista Latina de Comunicación Social, 65, 13.

Ospina Zapata, G. (2012, 18 de agosto). Nada para mis Biblioburros, dice Luis. Recuperado de http://www.elcolombiano.com/historico/nada_parara_mis_biblioburros_dice_luisOFEC_202783

Pereira, G. (2009). Preferencias adaptativas como bloqueo de la autonomía. En A. Cortina y G. Pereira, Pobreza y libertad: erradicar la pobreza desde el enfoque de Amartya Sen. Madrid: Tecnos.

PNUD (2010). Informe regional sobre desarrollo humano para América Latina. Santiago.

Ramonet, I. (1997). Un mundo sin rumbo. Madrid: Debate.

Ramos, F. (2013). El “tabú” periodístico de la monarquía en España. La crisis real y la crisis coyuntural. Revista Latina de Comunicación Social, 68, 217-247. Recuperado de http://www. revistalatinacs.org/068/paper/975_ Vigo/09_Ramos.html

Scolari, C. (2008). Hipermediaciones, elementos para una teoría de la comunicación digital interactiva. Barcelona: Gedisa.

Semana (2013, 12 de marzo). Vergüenza: Colombia entre los peores en educación. Recuperado de http:// www.semana.com/nacion/articulo/ colombia-entre-ultimos-puestosprueba-pisa/366961-3

Sen, A. (2000). Desarrollo y libertad. Barcelona: Planeta.

Sunkel, G., Trucco, D. y Möller, S. (2011). Aprender y enseñar con las tecnologías de la información y las comunicaciones en América Latina: potenciales beneficios. Cepal.

Toffler, A. (1981). La tercera ola. México: Edivisión.

Carmona, J. O. I. (2008). El prosumidor. El actor comunicativo de la sociedad de la ubicuidad. Palabra Clave, 11(1).

Unesco (1984). La educación en materia de comunicación. París: Unesco.

Wilson, C. y Duncan, B. (2009). La implementación de programas de educación en medios: el caso Ontario. Comunicar, 32, 97-107. 
WVS (2012). World Values Survey Zuleta, E. (2000). El elogio de la di2012: The world's most comprehen- ficultad. Recuperado de http://www. sive investigation of political and so- utp.edu.co/rectoria/documentos/elciocultural change. Recuperado de elogio-de-la-dificultad http://www.worldvaluessurvey.org 